\title{
AN EXPERIMENTAL AND NUMERICAL METHOD TO INVESTIGATE THE OXIDE BEHAVIOR IN CORROSION OF REINFORCED CONCRETE
}

\author{
BEATRIZ SANZ $*$, JAIME PLANAS ${ }^{*}$ AND JOSÉ M. SANCHO ${ }^{\dagger}$ \\ *Universidad Politécnica de Madrid, ETSI Caminos, Canales y Puertos, Profesor Aranguren 3, Madrid, Spain \\ e-mail: beatriz.sanz@upm.es, jaime.planas@upm.es \\ † Universidad Politécnica de Madrid, ETS de Arquitectura, Avda. Juan de Herrera 3, Madrid, Spain \\ e-mail: jose.sancho@upm.es
}

Key words: Finite Element Modeling, Cohesive Crack, Reinforced Concrete, Accelerated Corrosion, Fracture Mechanics

\begin{abstract}
In the prediction of corrosion-induced cracking of reinforced concrete, the mechanical behavior of the oxide has been proved to be crucial. However, there is a lack of experimental information, and the values of the oxide parameters have to be assumed in the models. In this work, a methodology is presented for indirect determination of the mechanical parameters of the oxide layer. It combines the results from accelerated corrosion tests, carried out in concrete prisms reinforced with a tube equipped with special instruments, and numerical simulations using a model that reproduces the cohesive fracture of concrete and the expansive behavior of the oxide. From the joint analysis of the experimental and numerical results, relevant conclusions about the oxide behavior have been disclosed, and values for the oxide parameters are proposed. With those, the experimental results reported by other authors have been simulated, with satisfactory results, which supports the model.
\end{abstract}

\section{INTRODUCTION}

Corrosion of the reinforcement occurs when depassivating substances go through the cover and break the passivity of steel. As a consequence, an oxide layer is generated around the steel bars, which results in a decrease in the cross-sectional area of the reinforcement, loss of bond, and cracking of the cover, among other effects [1], thus, reducing the service life of the structure. Focusing on cracking of the cover, many works have contributed to a better understanding of the factors affecting that [1-3], and models have been developed to simulate that phenomenon, from Bazant's physical model [4] and the pioneering finite element model by Molina et al [5] to models with discrete cracking, as the one used in this work [6]. In the simulation of corrosion-induced cracking, the oxide parameters have been proved to be essential. However, there is a lack of experimental information about those parameters, due to the difficulty to perform direct measurements at the oxide layer, and their values have to be assumed in the models. In particular, the expansion ratio controls the volume of the oxide layer, which can range from 2 to 6.5 times that of the initial steel [7], depending on the type of oxide generated, which is actually an unknown. Another influencing parameter is the shear stiffness, which affects the crack pattern of the specimens, as assessed in previous works [8], and for which no experimental information was found. Regarding the elastic modulus, a wide range of values has been reported in the literature [9, 10], which could be explained mainly by the different conditions in the tests, by the simplifications in the models, and by the results being inferred from measurements with low sensitivity to variations in those parameters. 
To get further knowledge about the oxide behavior and to obtain information close to the oxide layer, a novel set-up for accelerated corrosion tests was designed using as specimens concrete prisms reinforced with a steel tube [11]. From the experiments, information was provided regarding the variation of inner volume and inner diameter of the tube, which were measured using special instruments, the width of the main crack, which develops at the cover, and the final crack pattern of the specimens. In parallel, a model was developed that combines elements with an embedded crack [12], which reproduce concrete fracture following the standard cohesive model [13], and expansive joint elements, which reproduce the expansive behavior of the oxide [6]. Simulations of the tests showed that the curves of measurements are very sensitive to variations in the model parameters, which is essential for indirect determination of the mechanical parameters of the oxide.

In this work, a method to determine the oxide parameters is presented, based on the joint analysis of the experimental results and those from a numerical study, and values for the oxide parameters are proposed that could be used as a reference in the models from other authors. For further support of the method, the experimental results reported by other authors, in particular those from Andrade et al [1] and from Maaddawy and Soudky [3], have been simulated using the model and the oxide parameters proposed in this work, with satisfactory results.

This paper is organized as follows: Sec. 2 overviews the main aspects of the method, Sec. 3 presents and discuss the results, Sec. 4 shows application of the model to reproduce the experimental results reported by other authors, and Sec. 5]concludes this work.

\section{DESCRIPTION OF THE METHOD}

\subsection{Outline of the experiments}

The method proposed to determine the oxide parameters is based on experimental results of a previous work. For completeness of the text, the main aspects of the experiments are presented next, while the details can be found in [11,14].

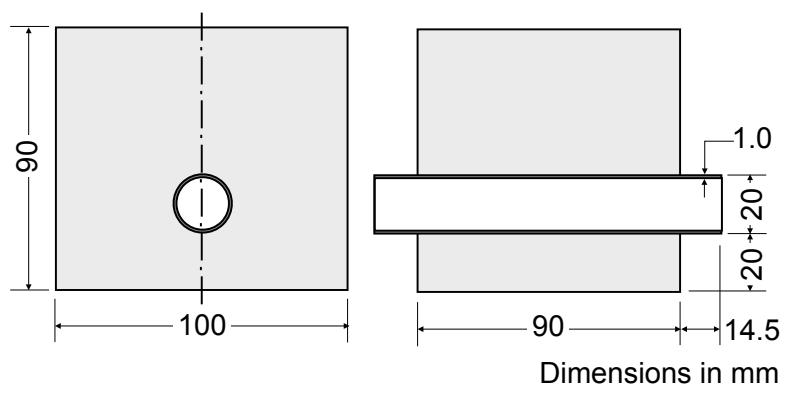

Figure 1 : Specimens for accelerated corrosion.

To gain more insight in the way the corrosion product interacts with the steel and the surrounding concrete, the specimens were concrete prisms reinforced with a steel tube, instead of a bar. Figure 1 shows a sketch with the geometry of the specimens. Those were designed to obtain a dominant mode of failure, consisting of a single main crack developing at the cover.

To fabricate the specimens, a relatively porous concrete was manufactured using Portland Cement I 52.5 R, with a water cement ratio of 0.5 and demineralized water. Silicious aggregate was used with a maximum size of $8 \mathrm{~mm}$, and calcium chloride was added to produce depassivation of the steel. Table 1 shows the mix proportions. Precision smooth steel tubes were used as reinforcement. Prior to their usage, the tubes were cleaned following the procedure described in [15], and an enamel coating was applied to both ends to avoid corrosion during casting and curing, while keeping a central portion of $80 \mathrm{~mm}$ in length free to corrode. For that study, eight reinforced prisms were casted from a single batch. After casting, the specimens were kept in the molds for $24 \mathrm{~h}$ in laboratory conditions, and then in a bath of lime saturated water at $20^{\circ} \mathrm{C}$ until testing time.

Accelerated corrosion tests were carried out in a temperature-controlled environment, with the specimen submerged in a water bath containing chlorides, as sketched in Fig. 2(a). To provide electrical insulation, an epoxy coating was applied to the concrete faces perpendicular to the tube, and the ends of the tube were protected with a PVC cap and a PVC ring. With those precautions, plane current flow and strain 
Table 1 : Concrete mix proportions, by weight of cement.

\begin{tabular}{ccccccc}
\hline Water & Cement & Sand & Aggregate & $\mathrm{CaCl}_{2}$ & Set Retarting & Superplasticizer \\
\hline 0.5 & 1 & 2.85 & 2.05 & 0.03 & 0.01 & 0.0013 \\
\hline
\end{tabular}

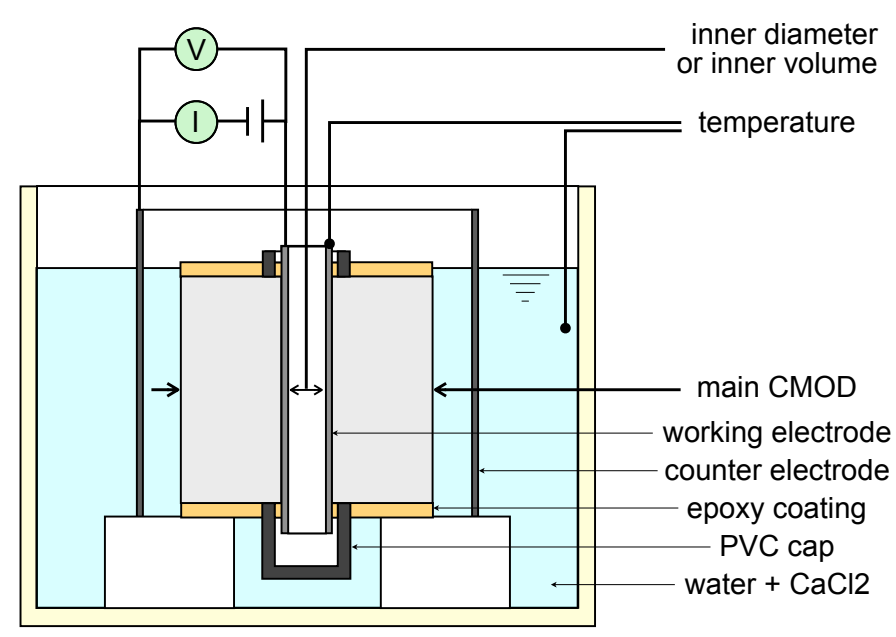

(a)

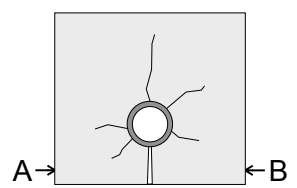

(b)

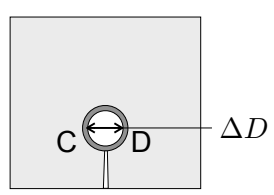

(c)

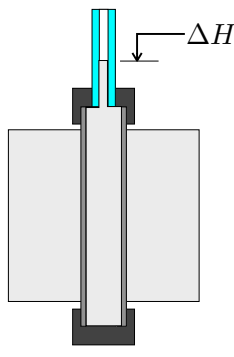

(d)

Figure 2 : Sketch of accelerated corrosion tests (a), and measurements of main CMOD (b), variation of inner diameter (c) and variation of capillary height (d).

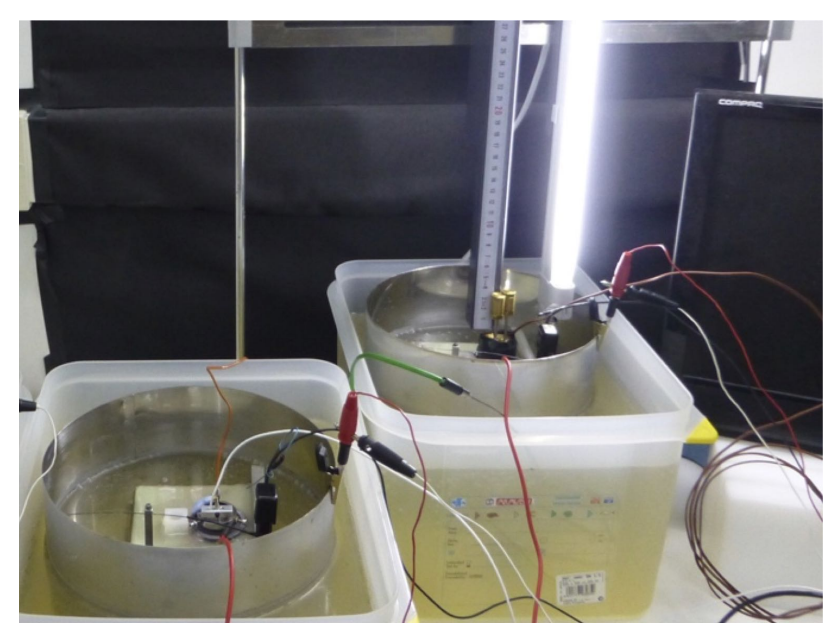

Figure 3 : View of accelerated corrosion tests.

were expected during the tests, resulting in nearly uniform corrosion along the length of the tube, which is essential for the numerical simulations. A stainless steel cylinder surrounding the specimen acted as the counter electrode. Figure 3 shows a picture of the tests. The tubes were corroded under a constant current, using the impressed current technique [1,3], with a density of current of $400 \mu \mathrm{A} / \mathrm{cm}^{2}$ for three days.

During accelerated corrosion, the current in- tensity $I$ and the electrical potential $V$ between the tube and the counter electrode were recorded using standard instruments, and the temperature of the tube and of the water bath were measured using type $\mathrm{K}$ thermocouples. A commercial extensometer adapted with extensions measured the width of the main crack at the middle section of the specimen, which is called main CMOD, as seen in Fig. 2(b). Finally, the specimens were instrumented either with a capillary circuit or with a diametral extensometer, which were specially designed to measure, respectively, the variation of inner volume and the variation of inner diameter of the tube. The variation of inner diameter was measured at the diameter perpendicular to the expected main crack, as sketched in Fig. 2(c). For the measure of inner volume, the tube was filled with liquid and sealed except for an exit through a glass capillary tube, Fig. 2(d); then variations of inner volume produce variations in the liquid height at the capillary $\Delta H$, which was manually tabulated from pictures taken at regular intervals. In particular, a reduction of $1.0 \mu \mathrm{m}$ in the inner diameter of the steel tube would re- 


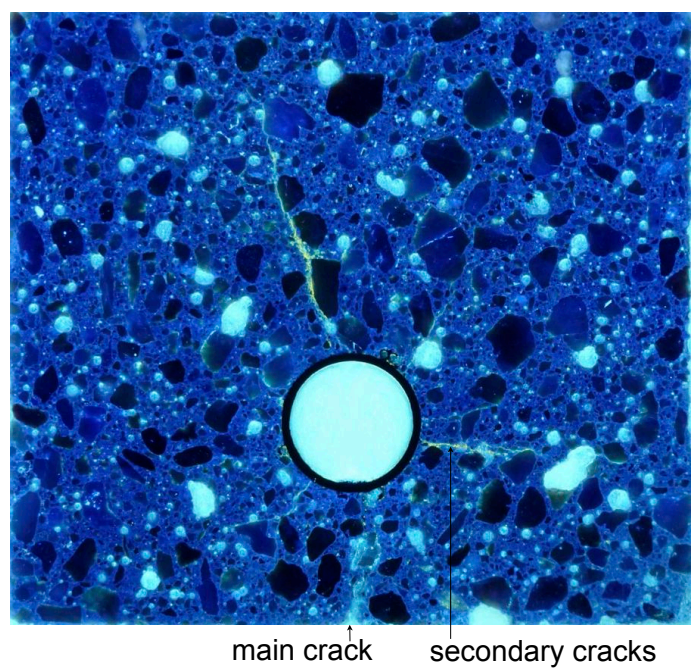

Figure 4: Crack pattern observed in corroded specimens.

sult in an increment of $11.16 \mathrm{~mm}$ in the liquid level, assuming uniform deformation along the free corroding length of the tube.

After corrosion, the loss of weight of the tubes was measured according to the ASTM G1 standard [16], and was compared with the theoretical loss calculated according to Faraday's law. The differences were within the ranges reported by other authors [2]. Thus, Faraday's law was demonstrated to be applicable, and from the record of electrical current, the corrosion depth $x$, defined as the amount of steel transformed into oxide, was calculated at each time, assuming $100 \%$ of efficiency of the current and uniform corrosion along the length of the tube.

Finally, the crack pattern was studied in slices of the specimens. Those were impregnated under vacuum, following the method described in [6], to enhance crack visibility under ultraviolet light. Normally, the pattern was analyzed at four different cross-sections per specimen. In all the specimens, a main crack was observed at the cover, and several thin secondary cracks surrounding the tube. Fig. 4 shows an example of the pattern observed in one of the slices. Notice that the main crack is less bright than the other cracks, due to being filled with oxide [6].

Complementarily, specimens were fabricated from the same batch and kept in the same conditions, for mechanical characterization of concrete. In particular, six cylinders $150 \mathrm{~mm}$ in diameter and $300 \mathrm{~mm}$ in height, and four beams with a cross-section of $100 \times 100 \mathrm{~mm}^{2}$ and $500 \mathrm{~mm}$ in length were casted. With those, the following tests were conducted at the age of 28 days: three compression tests, following the ASTM C39 standard [17], to determine compressive strength of concrete $f_{c}$; three brazilian tests, following the ASTM C496 standard [18] and some recommendations by Rocco [19], to determine the tensile strength of concrete $f_{t}$; and four three-point bending tests, following the method proposed by Planas et al [20], from which, in combination with the results of the brazilian tests, a bilinear approximation of the softening curve of concrete was calculated. The results of these tests were used as input for the concrete in the numerical simulations, and are shown in Sec. 2.3 for convenience of the text.

\subsection{Outline of the numerical model}

The model combines elements that reproduce fracture of concrete and elements that reproduce the oxide expansion as explained next.

Fracture of concrete is described according to the standard cohesive model, introduced by Hillerborg et al [13]. In that model, when a crack appears, it is assumed to transmit stresses at its faces, following a softening curve that depends on the crack width. To simplify the calculations, in this work a bilinear approximation of the curve was used. Fig. 5 shows the parameters defining it. That behavior is implemented in elements with an embedded adaptable crack [12], within the finite element framework COFE (Continuum Oriented Finite Element). Those are constant strain triangles with strong discontinuity kinematics that follow a central force model. In the elements, the crack path is calculated depending on the local stress field, and can reorient until a threshold opening $w_{t h}$ is reached, calculated as $w_{t h}=\alpha^{\prime} w_{1}$, where $\alpha^{\prime}$ is an adaption factor equal to 0.2 [12], and $w_{1}$ is defined in Fig. 5 .

To simulate the oxide action, expansive joint elements were used [6], which are interface elements with zero initial thickness that reproduce 


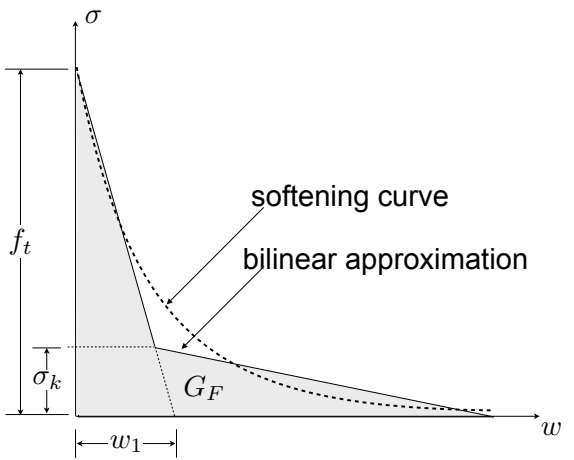

Figure 5 : Parameters that define the bilinear approximation of the softening curve, where $f_{t}$ is the tensile strength of concrete, $w_{1}$ the horizontal intercept of the first brach of the curve with the abscisas axis, $G_{F}$ the fracture energy, and $\sigma_{k}$ the stress at the break in the curve.

the volumetric expansion of oxide and its mechanical behavior. The volumetric expansion is calculated as $\beta x$, where $\beta$ is the expansion factor, which depends on the specific volume of steel $v_{\text {st }}$ and oxide $v_{\text {ox }}$ as

$$
\beta=\frac{v_{\mathrm{ox}}}{v_{\mathrm{st}}}-1
$$

To reproduce a fluid-like behavior, as proposed by Molina et al [5], debonding and separation are implemented in the element, which are achieved, respectively, by introducing a shear stiffness $k_{t}$ much less than the normal stiffness $k_{n}$, and by a directionality factor $\eta$ that diminishes the normal stiffness in tension. For a given mechanical displacement $\mathbf{w}$, the traction vector $\mathbf{t}$ acting on the element is calculated as:

$$
\mathbf{t}=\eta k_{n}(\mathbf{w} \cdot \mathbf{n}-\beta x) \mathbf{n}+k_{t}[\mathbf{w}-(\mathbf{w} \cdot \mathbf{n}) \mathbf{n}]
$$

where $\mathbf{n}$ is the unit normal to the element, and no stresses appear for a free expansion of the oxide. It is important to notice that the joint element only comprehends the increment of volume of the oxide, while the steel section is kept constant, to avoid re-meshing, as sketched in Fig. 6, thus, fictitious properties of the element are calculated depending on the actual steel and oxide properties to maintain mechanical equivalence of the real and simulated systems, which turn out to be inversely proportional to the corrosion depth. To avoid numerical instabilities, numerical cutoffs $k_{n}^{0}$ and $k_{t}^{0}$ were proposed for

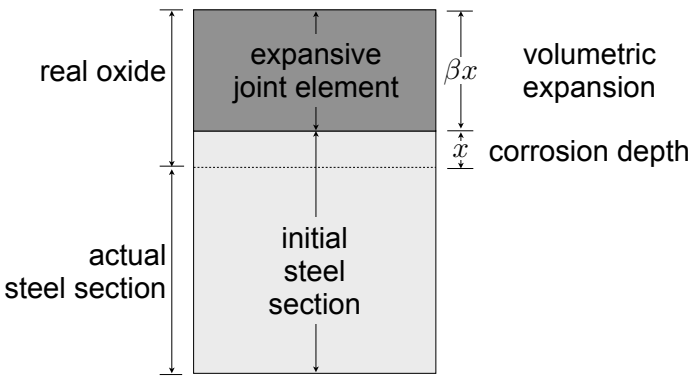

Figure 6 : Sketch of the expansive joint element.

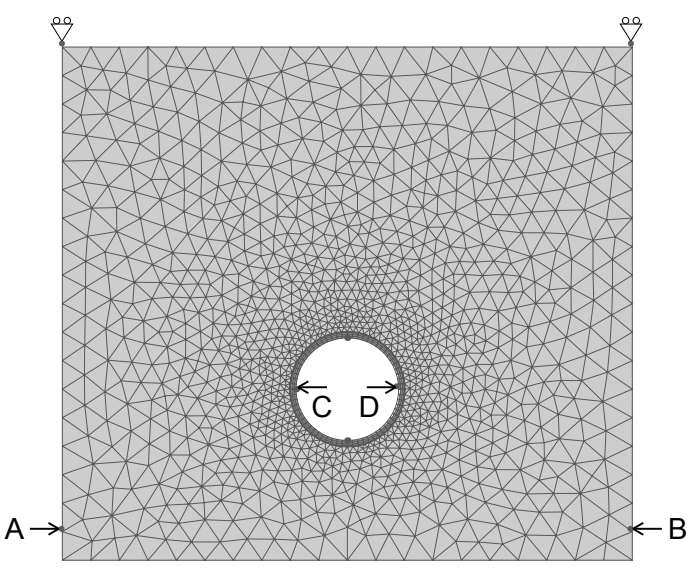

Figure 7 : Mesh used in the simulations.

a small corrosion depth $x_{0}$, and, thus, the normal stiffness is calculated as

$$
\begin{array}{ll}
k_{n}=k_{n}^{0} \approx \frac{N_{\text {ox }}}{(1+\beta) x_{0}}, & \text { if } x \leq x_{0} \\
k_{n}=k_{n}^{0} \frac{x_{0}}{x}, & \text { if } x>x_{0}
\end{array}
$$

where $N_{o x}$ is the normal modulus of the oxide, which coincides with its bulk modulus if the oxide is assumed to behave like a liquid, and a similar expression results for the shear stiffness replacing $N_{\text {ox }}$ by the shear modulus $G_{\text {ox }}$.

\subsection{Simulations and parametric study}

Simulations were carried out using twodimensional models of the specimens, with a tube as a reinforcement, using the mesh shown in Fig. 7. The mesh was generated using the program Gmsh [21]. The steel was modeled with quadrilateral elements with assumed enhanced strain, the concrete with constant strain triangles with an embedded crack, and the oxide with four-node expansive joint elements. The number of elements of steel was 16 per 
Table 2 : Mechanical parameters of the materials in reference simulations, where $E$ is the elastic modulus, $\nu$ Poisson's ratio, $f_{t}$ the tensile strength, $G_{F}$ the fracture energy, $w_{1}$ the horizontal intercept with the abscissas axis, $\sigma_{k}$ the stress at the break point in the softening curve, $\alpha^{\prime}$ the adaption factor of the crack, $\beta$ the volumetric expansion factor, $x_{0}$ the cutoff corrosion depth, $k_{n}^{0}$ and $k_{t}^{0}$ the cutoff normal and shear stiffnesses and $\eta$ the reduction factor of the tensile stiffness.

\begin{tabular}{rccccccc}
\hline & $E(\mathrm{GPa})$ & $\nu$ & $f_{t}(\mathrm{MPa})$ & $G_{F}(\mathrm{~N} / \mathrm{mm})$ & $w_{1}(\mathrm{~mm})$ & $\sigma_{k}(\mathrm{MPa})$ & $\alpha^{\prime}$ \\
\hline Steel & 200 & 0.3 & - & - & - & - & \\
Concrete: linear & 36.1 & 0.2 & 2.78 & 0.0597 & 0.0430 & - & 0.2 \\
Concrete: bilinear & 36.1 & 0.2 & 2.78 & 0.107 & 0.0430 & 0.322 & 0.2 \\
& $\beta$ & $x_{0}(\mathrm{~mm})$ & $k_{n}^{0}\left(\mathrm{~N} / \mathrm{mm}^{3}\right)$ & $k_{t}^{0}\left(\mathrm{~N} / \mathrm{mm}^{3}\right)$ & $\eta$ & & \\
\hline Oxide: initial & 1.0 & $1.0 \times 10^{-3}$ & $1.0 \times 10^{6}$ & $1.0 \times 10^{-14}$ & $1.0 \times 10^{-11}$ & \\
\hline
\end{tabular}

quarter of circumference and 3 at the thickness of the tube, which in previous works was found to be adequate to capture bending [11]; The surrounding elements of concrete and oxide matched that mesh, and for the concrete, the size was linearly increased to be five times larger at the edges. The boundary conditions were two simple supports.

Table 2 shows the value of the parameters of the materials in reference simulations. The steel was modeled as linear elastic, assuming standard values. The concrete followed a cohesive behavior, for which the parameters determined in mechanical tests were used. In preliminary simulations in which cases with small crack widths are studied, a linear approximation of the softening curve was used to speed up the computations; then, for accurate calculations, a bilinear curve was used. For the oxide, the parameters used in [6] were used as a reference, which are based on a fluid-like behavior and an expansion factor $\beta=1.0$ as suggested in [5], a vanishingly small shear modulus and free separation. The cutoff stiffnesses were calculated for a corrosion depth $x_{0}=1 \mu \mathrm{m}$, which was adequate to maintain the accuracy of the computations while keeping them stable.

A study was carried out to analyze the influence of the model parameters on the curves of results, modifying one parameter at each time while maintaining the remaining parameters with the values indicated in Table 2. The expansion factor and the shear stiffness were identified as the most influencing parameters. Then those two parameters were modified to fit
Table 3 : Range of values to determine the best values for the parameters of the expansive joint element, where $\beta$ is the expansion factor and $k_{t}^{0}$ is the cutoff shear stiffness.

\begin{tabular}{cl}
\hline Parameter & Range of values \\
\hline$\beta$ & $1.8,1.9,2.0$ \\
$k_{t}^{0}\left(\mathrm{~N} / \mathrm{mm}^{3}\right)$ & $5 \times 10^{2}, 1.0 \times 10^{3}, 1.5 \times 10^{3}$ \\
\hline
\end{tabular}

the numerical results to the experimental ones within the ranges indicated in Table 3. First, the value of the expansion factor was modified, using linear softening to speed up the computations, and, second, the shear stiffness was modified, using bilinear softening.

The calculations were driven by the corrosion depth $x$, and the free radial expansion $\beta x$ was computed at each step. A total corrosion depth of $20 \mu \mathrm{m}$ was applied in 40 steps, which was enough to produce a stable pattern of cracks and to capture the crucial points in the curves of results, except for the study of the expansion factor $\beta$, in which the steps of $0.1 \mu \mathrm{m}$ were used for an accurate determination of that parameter.

From the simulations, the map of stresses and the crack pattern were analyzed, and the curves of main CMOD, variation of inner diameter and variation of inner volume of the tube, for which the displacement was recorded at the adequate points, as shown in Fig. 7.

\section{RESULTS AND DISCUSSION}

\subsection{Results of accelerated corrosion tests}

Figure 8 shows in light color the envelope and the average of the results obtained in accelerated corrosion tests, which are used as a reference to determine the oxide parameters. The 


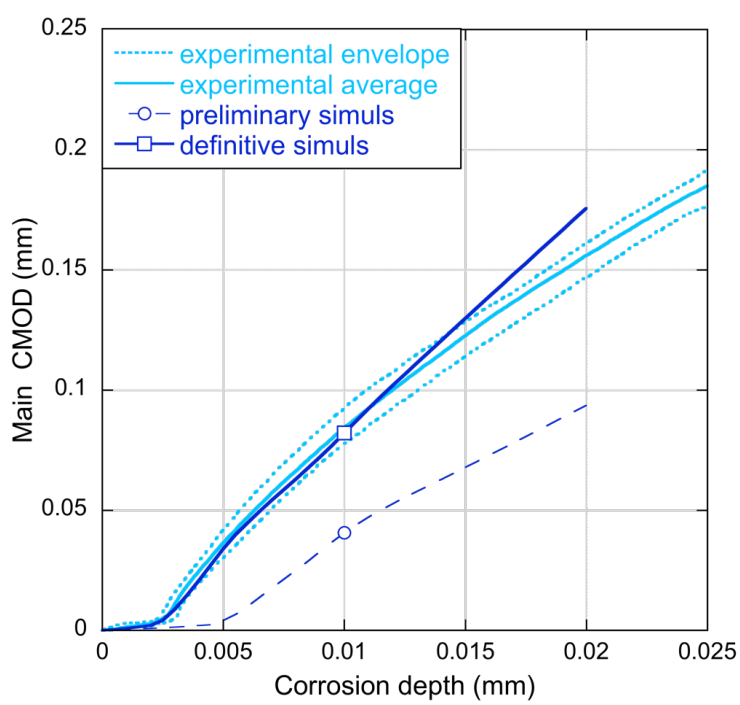

(a)

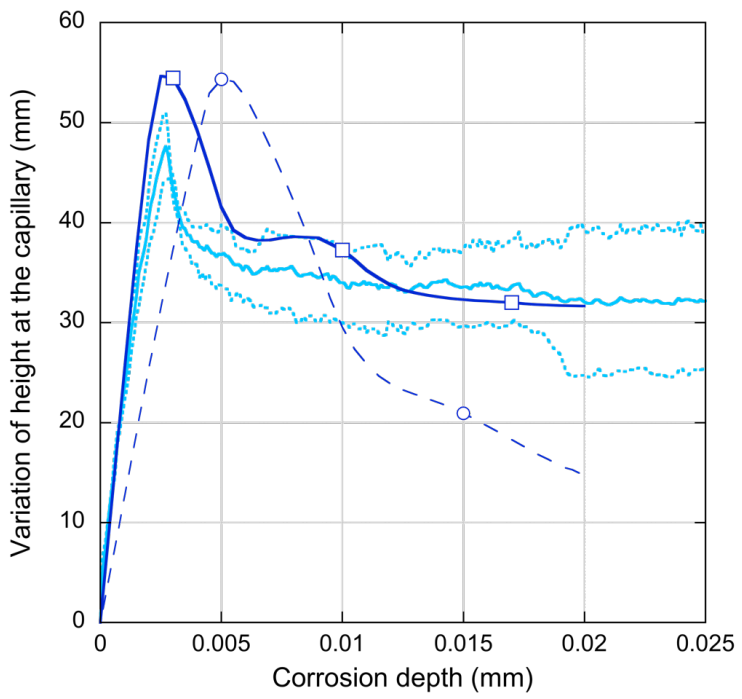

(b)

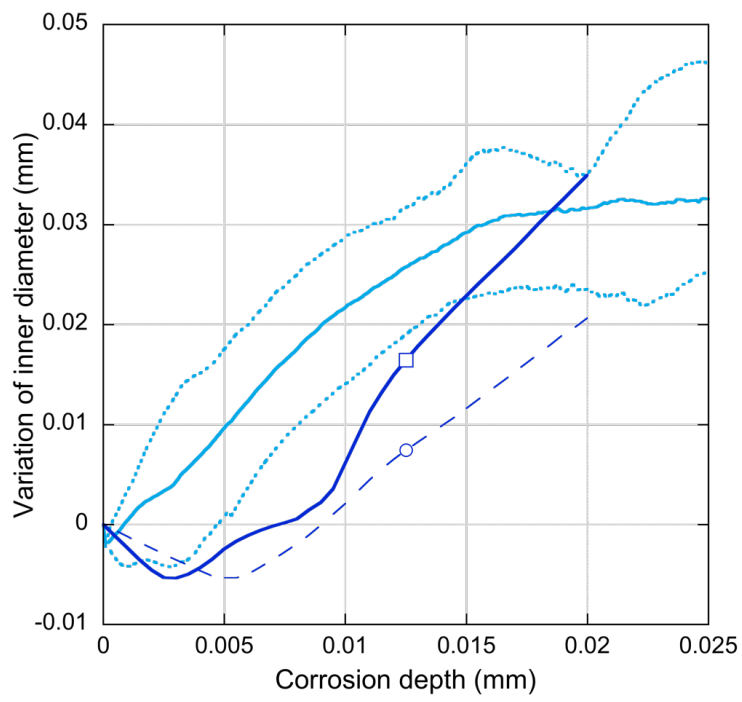

(c)

Figure 8 : Results of accelerated corrosion tests and numerical simulations: curves of main CMOD (a), variation of capillary height (b) and variation of inner diameter (c). main CMOD, Fig. 8(a), grew slowly for small corrosion depths, but then there was a break in the curves and the slope increased, which indicates development of the main crack. The curves of variation of capillary height, Fig. $8($ (b), had a step rise up to a peak that coincides with the corrosion depth of the break point in the curves of main CMOD, which was called critical corrosion depth, and then a descent; i.e., there was a diminution in the inner volume of the tube until development of the main crack, and then the volume partially recovered. Finally, the variation of inner diameter, Fig. 8(c) displayed an overall trend to grow, although there was a high scatter in this measurement. It should be noticed that the different trends observed in the curves of variation of inner volume and inner diameter indicates that the deformation of the tube was not uniform.

\subsection{Results of the numerical study}

The results of preliminary simulations carried out using the parameters shown in Table 2 are displayed in Fig. 8 by the dark-color dotted line. From those, it was assessed that the model captured the main trends observed in the experiments, with a break in the curves of main CMOD and a peak of capillary height occurring for a given corrosion depth. However, the numerical curves were delayed with respect to the experimental ones, revealing the need to modify the oxide parameters.

Thus, a parametric study was carried out to analyze the response of the curves of results for variations in the expansive joint element parameters, i.e., the normal stiffness $k_{n}^{0}$, the shear stiffness $k_{t}^{0}$, the directionality factor $\eta$ and the expansion factor $\beta$. It was seen that variations in the normal stiffness for $k_{n}^{0} \geq 1.0 \times$ $10^{6} \mathrm{~N} / \mathrm{mm}^{3}$ did not produce relevant changes in the results, while the expansion factor, the shear stiffness and the directionality factor had a strong effect. In particular, the expansion factor scales the results with the corrosion depth, since the volumetric expansion at each step is $\beta x$, thus, it controls the critical corrosion depth, and the shear stiffness governs the behavior af- 
ter the critical corrosion depth, i.e., after development of the main crack. The reduction factor had effects similar than those of the shear stiffness, although less pronounced, since it only affects the projection of the traction vector of neighbor elements.

Finally, the best values of the oxide parameters to reproduce the experimental results were determined as explained next. The normal compressive stiffness $k_{n}$ was maintained as initially, since higher values increase the number of iterations but does not modify the results. The reduction factor of the tensile stiffness was also maintained as initially, since it corresponds to a normal tensile stiffness of virtually zero, which is coherent with the hypothesis of a fluid-like behavior. Then the expansion factor and the shear stiffness were modified within the ranges displayed in Table 3. Since the curves of variation of inner diameter had the highest scatter, and to avoid the influence of the diameter on the measurements, we will mainly focus on the curves of main CMOD and variation of capillary height. The expansion factor was modified to fit the numerical critical corrosion depth to that in the experiments, as shown in Fig. 9 In this step, linear softening was used to speed up the computations, since the critical corrosion depth occurs for crack widths less than $6.0 \mu \mathrm{m}$, which, for these experiments, is much smaller than the horizontal intercept of the first brach of the bilinear curve $w_{1}$, as seen in Fig. 8(a) and Table 2. The best value was found to be $\beta=2.0$, which corresponds to a ratio of the specific volumes of oxide and steel of 3.0. Then the cutoff of the shear stiffness was modified to fit the tail of the curves, as shown in Fig. 10, using bilinear softening, as the tail corresponded to crack widths higher than $w_{1}$. In previous works, it was observed that a strongly reduced value of the shear stiffness led to a single crack that separated the section into two halves, while a too high value led to clamping of cracks and to stress concentration at the root of the cracks [8]; thus, it was increased although still kept much smaller than the normal stiffness. The best value was found to be $k_{t}^{0}=1000 \mathrm{~N} / \mathrm{mm}^{3}$.
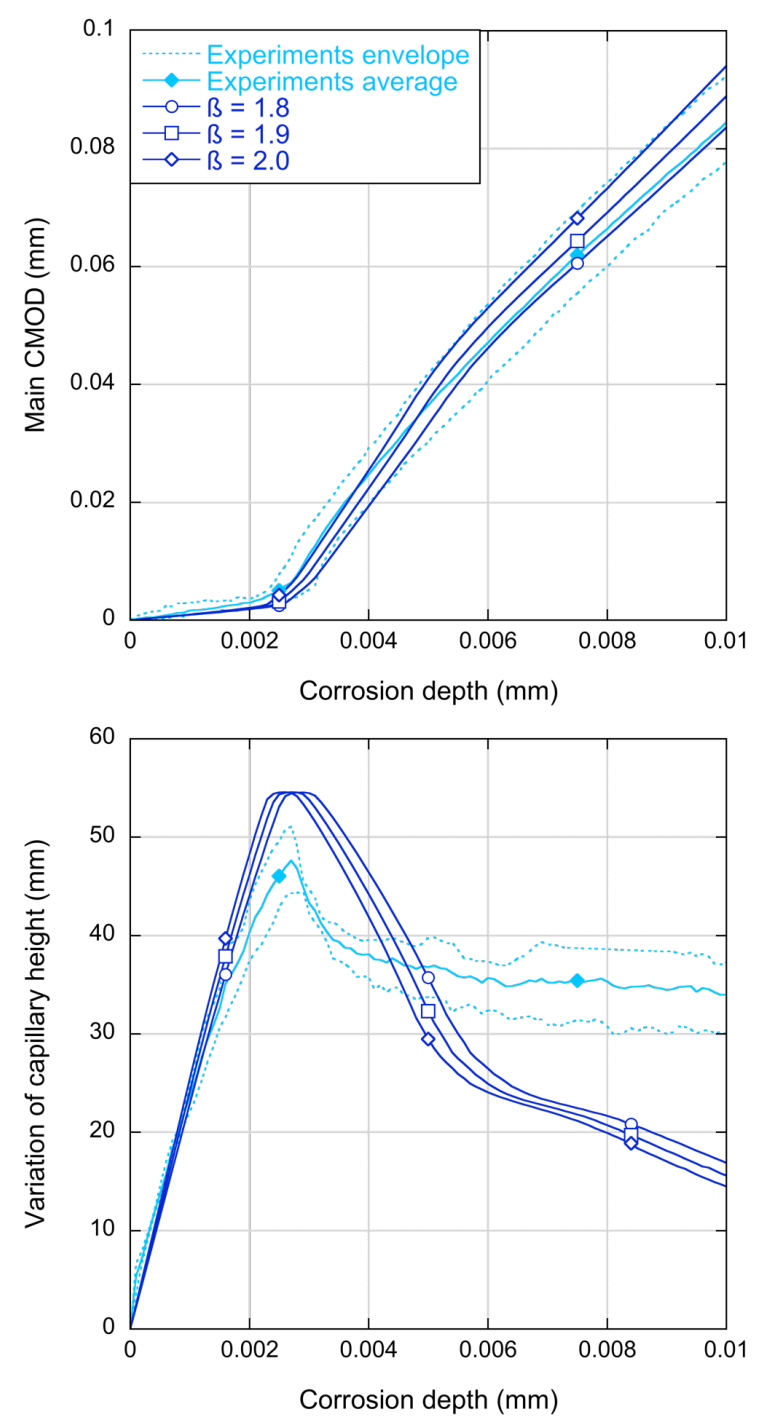

Figure 9 : Determination of the best value of the expansion factor $\beta$ to fit the experimental results.

It should be noticed that these stiffnesses correspond to the expansive joint element, not to the oxide itself, but they are related to the properties of oxide by Equation (3) and, so, for a expansion factor $\beta=2.0$, they are equivalent to a bulk modulus of oxide $K_{\mathrm{ox}}=3.0 \mathrm{GPa}$ in compression, a tensile modulus of virtually zero and a shear modulus $G_{\text {ox }}=3.0 \mathrm{MPa}$, i.e., 1000 times smaller than $K_{\mathrm{ox}}$, which is in accordance with the hyphothesis of a fluid-like behavior.

The results for definitive simulations of the tests with the oxide parameters determined in this study are displayed in Fig. 8 by the darkcolor solid line. The numerical curves properly reproduced the results, except for the final part 

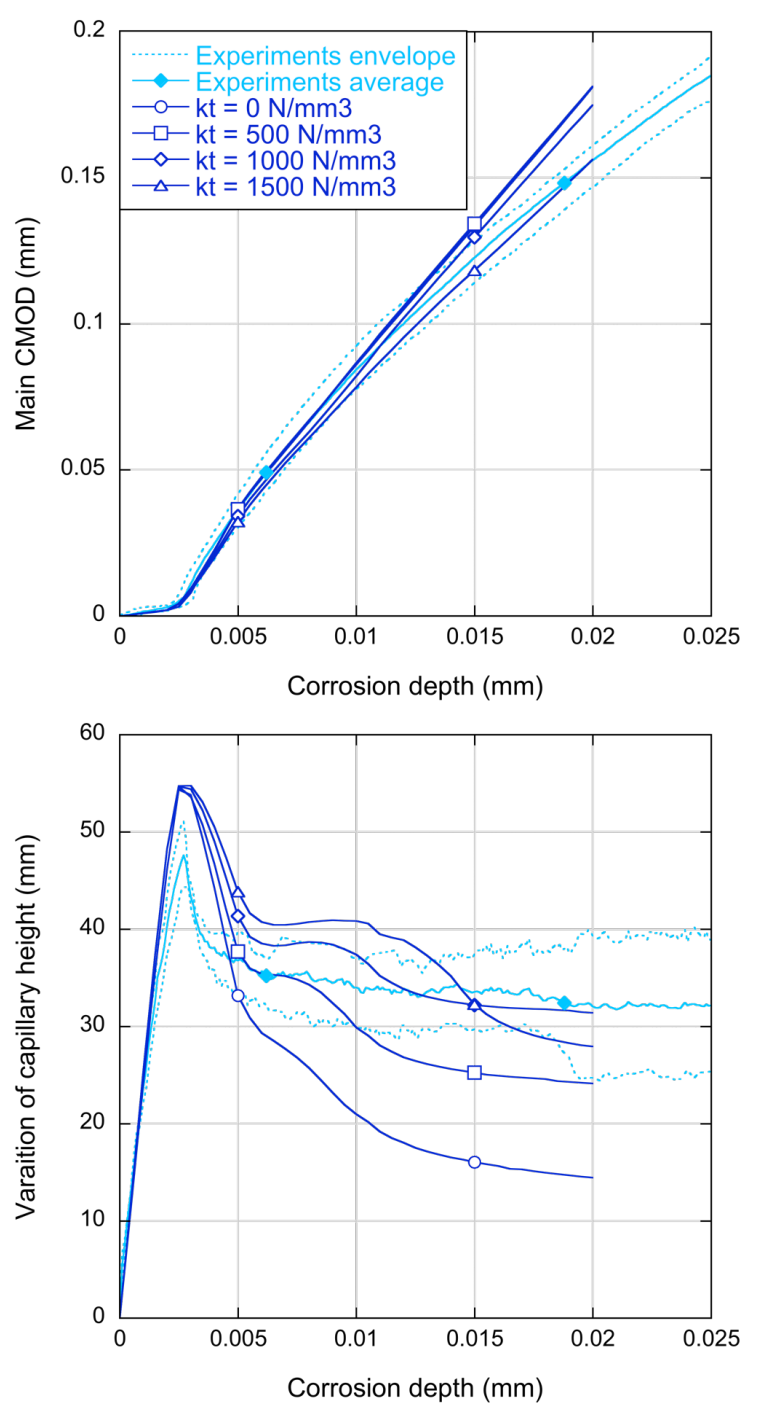

Figure 10 : Determination of the best value of the cutoff of the shear stiffness $k_{t}^{0}$ to fit the experimental results.

of the curves of main CMOD, the peak in the capillary height and the variation of inner diameter, due to some limitations in the model and in the experiments, as explained in [11], such as not taking into account diffusion of oxide within the cracks, a phenomenon pointed out in [5] and assessed in [6], not considering anisotropy of concrete neither non-uniform corrosion, and the different loading rate in the mechanical tests and in the corrosion tests, which might affect the fracture parameters of concrete [22, 23]. The simulated pattern, shown in Fig. 11, resembled the experimental one, with a main crack developing at the cover, and a number of thin secondary cracks surrounding the
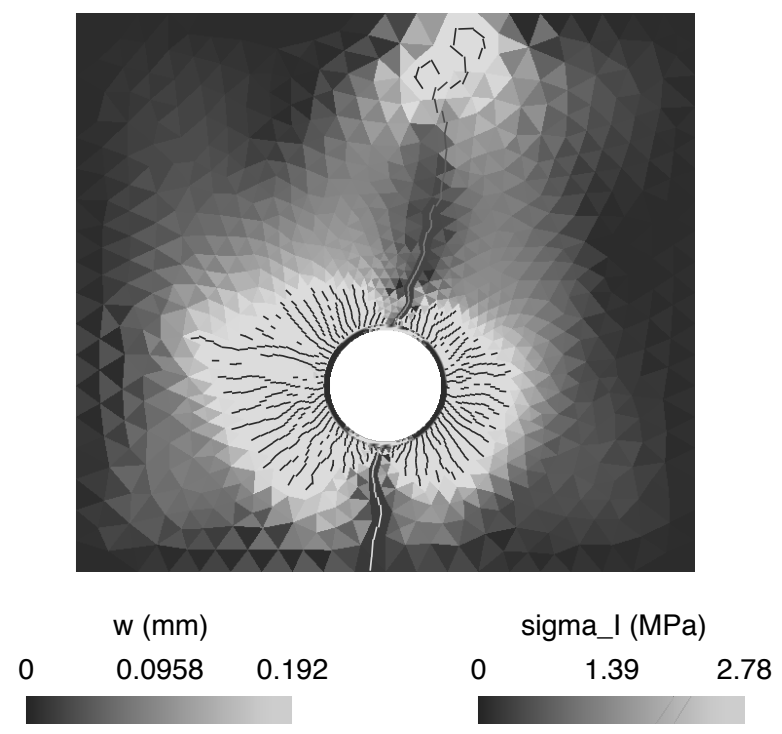

Figure 11 : Crack pattern obtained in simulations.

specimen within the ranges reported in [6].

\section{APPLICATION OF THE MODEL}

For further support of the method, simulations were carried out for two reference studies found in the literature, using the model and the values determined for the oxide parameters, which are summarized in Table 4 . In particular, the tests from Andrade et al [1] and Maaddawy and Soudki [3] were simulated. Meshes were generated for 2D FE models of the specimens as described in Sec. 2.3, except for the steel, which was modeled with constant strain triangles and with eight elements per quarter of circumference. A corrosion depth of $20 \mu \mathrm{m}$ was applied in 40 steps. The values for the steel were as those in Table 2. For the concrete, standard values were assumed, shown in Table 5, except for the tensile strength of Andrade tests, for which a value of $3.55 \mathrm{MPa}$ was reported.

Figure 12 shows the final crack pattern obtained in simulations of the tests from Andrade et al, for specimens type I, II and III defined in the paper. In all the cases, the simulations predicted a main crack whose position coincided with those reported in the paper, except for that of specimen I, which developed in a different side of concrete, due to the indeterminacy of a weakest face for the symmetry of the bar at that corner, as pointed out in [1]. 
Table 4 : Definitive values for the expansive joint element parameters, where $\beta, x_{0}, k_{n}^{0}, k_{t}^{0}$ and $\eta$ are defined in Table 2

\begin{tabular}{cccccc}
\hline & $\beta$ & $x_{0}(\mathrm{~mm})$ & $k_{n}^{0}\left(\mathrm{~N} / \mathrm{mm}^{3}\right)$ & $k_{t}^{0}\left(\mathrm{~N} / \mathrm{mm}^{3}\right)$ & $\eta$ \\
\hline Oxide: def. & 2.0 & $1.0 \times 10^{-3}$ & $1.0 \times 10^{6}$ & $1.0 \times 10^{3}$ & $1.0 \times 10^{-11}$ \\
\hline
\end{tabular}

Table 5 : Concrete parameters in simulations of the experiments presented in [1] and [3], where $E, \nu, f_{t}, G_{F}, w_{1}, \sigma_{k}$ and $\alpha^{\prime}$ are defined in Table 2 .

\begin{tabular}{lccccccc}
\hline & $E(\mathrm{GPa})$ & $\nu$ & $f_{t}(\mathrm{MPa})$ & $G_{F}(\mathrm{~N} / \mathrm{mm})$ & $w_{1}(\mathrm{~mm})$ & $\sigma_{k}(\mathrm{MPa})$ & $\alpha^{\prime}$ \\
\hline Concrete & 30.0 & 0.2 & $3.00 *$ & 0.100 & 0.03 & 0.4 & 0.2 \\
\hline *A value of $3.55 \mathrm{MPa}$ was used in the simulations of the tests from Andrade et al [1]
\end{tabular}

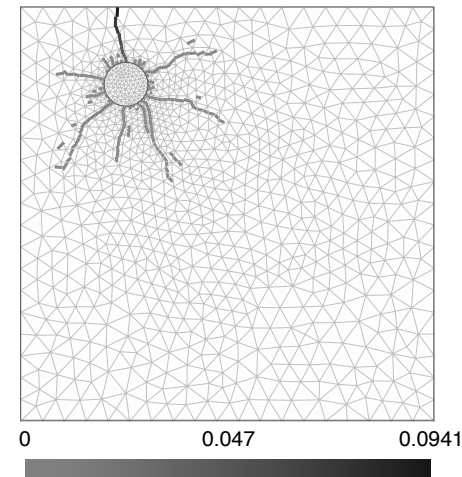

(a)

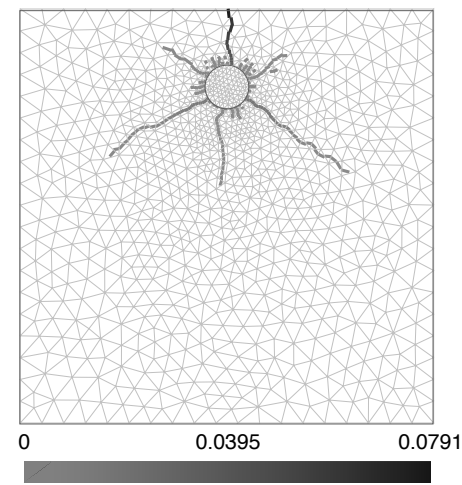

(b)

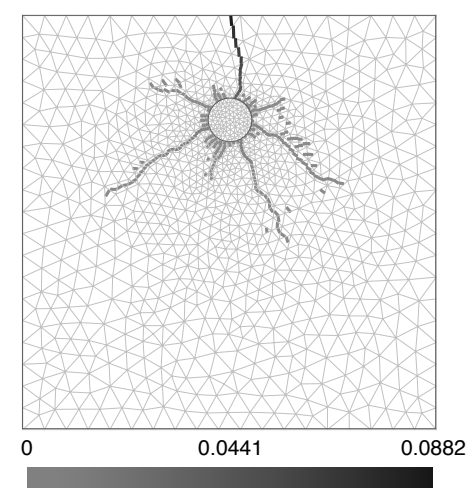

(c)

w (mm)

Figure 12: Map of cracks obtained in simulations of the tests from Andrade et al [1], for specimens type I (a), II (b) and III (c).
Nevertheless, a crack width of about $0.1 \mathrm{~mm}$ was reported for that specimen for a corrosion depth of $20 \mu \mathrm{m}$, which is in agreement with that in the simulations. Secondary cracks were reported at $4.30 \mathrm{~h}$ for specimen $\mathrm{I}$, and at $4.30 \mathrm{~h}$ and $7.30 \mathrm{~h}$ for specimens II and III, in simulations of the tests by Molina et al [5] using a smeared crack model, which is in accordance with the widest secondary cracks predicted in this work. It should be noticed that small differences in the crack width could be found with respect to those reported in the experiments, which could be explained by the oxide parameters being inferred from the results of specimens corroded with $400 \mu \mathrm{A} / \mathrm{cm}^{2}$, while specimens I to III in that work were corroded with $100 \mu \mathrm{A} / \mathrm{cm}^{2}$, and the current density affects the type of oxide generated [2]. Further work is in progress to analyze the influence of the density of current on the mechanical properties of the oxide.

Simulations were carried out for the experiments from Maaddawy and Soudky, carried out with a density of current of $350 \mu \mathrm{A} / \mathrm{cm}^{2}$, which is closer to that of the experiments in this work. Fig. 13 shows the final pattern obtained in the simulations. In the figure, only the cracks wider than $0.001 \mathrm{~mm}$ are shown, for not plotting cracks with an opening near to zero that appear in the calculations and close when dominant cracks develop, since the paper only reports information about visible cracks. The simulated crack pattern resembled the pattern type II defined in the paper, consisting of two surface cracks, one of them at the right side, and the other at the top surface of concrete, and a secondary crack joining the bars. 


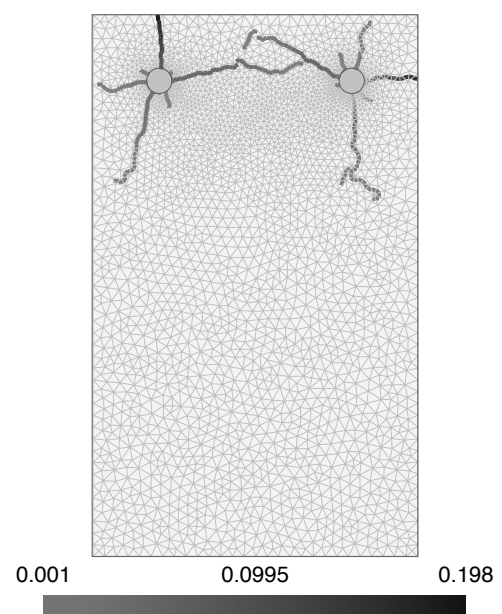

Figure 13 : Map of cracks obtained in simulations of the tests from Maaddawy and Soudky [3].

\section{CONCLUSIONS}

A method for indirect determination of the mechanical parameters of the oxide developed in corrosion of reinforced concrete has been presented, which combines the results of accelerated corrosion tests and numerical simulations. The specimens had a tube as reinforcement, instead of a bar, which provided additional mechanical information during the tests that resulted to be crucial in this work.

From the joint analysis of the experimental and numerical results, essential aspects were disclosed about the oxide behavior and values have been obtained for the oxide parameters for specimens corroded with $400 \mu \mathrm{A} / \mathrm{cm}^{2}$, which could be used in the models from other authors.

Simulations of reference experimental results found in the literature have been carried out using the proposed values for the oxide, with satisfactory results, which supports the method presented in this study.

\section{ACKNOWLEDGEMENTS}

The authors gratefully acknowledge the Secretaría de Estado de Investigación, Desarrollo e Innovación of the Spanish Ministerio de Economía y Competitividad for providing financial support for this work under the project BIA2014-54916-R.

\section{REFERENCES}

[1] C. Andrade, M.C. Alonso, and F.J. Molina. Cover cracking as a function of bar corrosion: Part i - experimental test. Materials and Structures, 26(8):453-464, 1993.

[2] M.C. Alonso, C. Andrade, J. Rodríguez, and J.M. Díez. Factors controlling cracking of concrete affected by reinforcement corrosion. Materials and Structures, 31(7):435-441, 1998.

[3] T. El Maaddawy and K. Soudki. Effectiveness of impressed current technique to simulate corrosion of steel reinforcement in concrete. Journal of Materials in Civil Engineering, 15(1):41-47, 2003.

[4] Z.P. Bazant. Physical model for steel corrosion in concrete sea structures- theory. Journal Structural Division, ASCE, 105:1137-1153, 1979.

[5] F.J. Molina, M.C. Alonso, and C. Andrade. Cover cracking as a function of bar corrosion: Part ii - numerical model. Materials and Structures, 26(9):532-548, 1993.

[6] B. Sanz, J. Planas, and J. M. Sancho. An experimental and numerical study of the pattern of cracking of concrete due to steel reinforcement corrosion. Engineering Fracture Mechanics, 114:26-41, 12 2013.

[7] K. Tuutti. Corrosion of steel in concrete, volume 4.82 of CBI Forskning 82:4. Swedish Cement and Concrete Research Institute, 1982.

[8] B. Sanz, J. Planas, and J.M. Sancho. Study of concrete cracking during accelerated corrosion tests in reinforced concrete. In J. G. M. Van Mier, G. Ruiz, C. Andrade, R.C. Yu, and X.X. Zhang, editors, Proceedings of the VIII International Conference on Fracture Mechanics of Con- 
crete and Concrete Structures FraMCoS8, Toledo, Spain, pages 2019-2028, 2013.

[9] S. Caré, Q. T. Nguyen, V. L'Hostis, and Y. Berthaud. Mechanical properties of the rust layer induced by impressed current method in reinforced mortar. Cement and Concrete Research, 38(8-9):1079-1091, 82008.

[10] B. J. Pease, A. Michel, A.E.A. Thybo, and H. Stang. Estimation of elastic modulus of reinforcement corrosion products using inverse analysis of digital image correlation measurements for input in corrosion-induced cracking model. In 6th International Conference on Bridge Maintenance, Safety and Management (IAMBAS). Lake Como, Italy, 72012.

[11] B. Sanz, J. Planas, and J.M. Sancho. A closer look to the mechanical behavior of the oxide layer in concrete reinforcement corrosion. International Journal of Solids and Structures, 62:256 - 268, 2015.

[12] J. M. Sancho, J. Planas, D. A. Cendon, E. Reyes, and J. C. Galvez. An embedded cohesive crack model for finite element analysis of concrete fracture. Engineering Fracture Mechanics, 74(1-2):7586, 2007.

[13] A. Hillerborg, M. Modéer, and P.E. Petersson. Analysis of crack formation and crack growth in concrete by means of fracture mechanics and fracture elements. Cement and concrete research, 6(6):773781, 1976.

[14] B. Sanz. Experimental and numerical study of cracking of concrete due to reinforcement corrosion. $\mathrm{PhD}$ thesis, Universidad Politécnica de Madrid, 2014.

[15] I. Kayafas. Technical note: Corrosion product removal from steel fracture surfaces for metallographic examination. Corrosion, NACE, 36(8):443-445, 81980.
[16] ASTM, editor. Standard Practice for Preparing, Cleaning and Evaluating Corrosion Test Specimens, number G 103. ASTM International, West Conshohocken, PA, 2003.

[17] ASTM, editor. Standard Test Method for Compressive Strength for Cylindrical Concrete Specimens, number C 3986. ASTM International, West Conshohocken, PA, 1986.

[18] ASTM, editor. Standard Test Method for Splitting Tensile Strength of Cylindrical Concrete Specimens, number C 496-90. ASTM International, West Conshohocken, PA, 1990.

[19] C. Rocco. Influencia del tamaño y mecanismos de rotura del ensayo de compresión diametral. $\mathrm{PhD}$ thesis, E.T.S.I. Caminos, Canales y Puertos, U.P.M., 1996.

[20] J. Planas, G. V. Guinea, J. C. Galvez, B. Sanz, and A. M. Fathy. Report 39: Experimental Determination of the StressCrack Opening Curve for Concrete in Tension - Final report of RILEM Technical Committee TC 187-SOC, chapter 3. Indirect tests for stress-crack opening curve, pages 13-29. RILEM Publications SARL, 2007.

[21] C. Geuzaine and J.-F. Remacle. Gmsh: a three-dimensional finite element mesh generator with built-in pre- and postprocessing facilities. International Journal for Numerical Methods in Engineering, 79(11):1309-1331, 2009.

[22] Z. P. Bazant and R. Gettu. Rate effects and load relaxation in static fracture of concrete. ACI Materials Journal, 89(5):456468, 1992.

[23] Z.P. Bazant and J. Planas. Fracture and size effect in concrete and other quasibrittle materials. C.R.C. Press, Boca Raton, F.L., 1998. 